\title{
Efficacy of DOPE/DC-cholesterol liposomes and GCPQ micelles as AZD6244 nanocarriers in a 3D colorectal cancer in vitro model
}

\begin{abstract}
Aim: In this work, we use cationic organic nanocarriers as chemotherapy delivery platforms and test them in a colorectal cancer 3D in vitro model. Materials \&

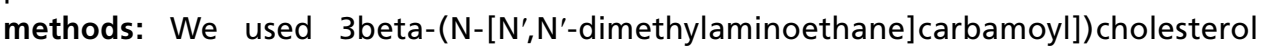
(DC-chol) and dioleoylphosphatidylethanolamine (DOPE) liposomes and N-palmitoyl$\mathrm{N}$-monomethyl-N,N-dimethyl-N,N,N-trimethyl-6-O-glycolchitosan (GCPQ) micelles, to deliver AZD6244, a MEK inhibitor, to HCT116 cells cultured as monolayers and in 3D in vitro cancer models (tumoroids). Results: Nanoparticle-mediated drug delivery was superior to the free drug in monolayer experiments and despite their therapeutic effect being hindered by poor diffusion through the cancer mass, GCPQ micelles were also superior in tumoroids. Conclusion: These results support the role of nanoparticles in improving drug delivery and highlight the need to include 3D cancer models in early phases of drug development.
\end{abstract}

First draft submitted: 21 June 2015; Accepted for publication: 4 December 2015; Published online: 20 January 2016

Keywords: 3D culture $\bullet$ artificial cancer mass $\bullet$ AZD6244 $\bullet$ chemotherapy • colorectal cancer $\bullet$ drug delivery $\bullet$ liposomes $\bullet$ micelles $\bullet$ tumoroids

Colorectal cancer is the third most frequent cancer and ranks fourth for cancer-related deaths in the world [1]. Chemotherapy is used as an adjuvant treatment after patients undergo surgery but both therapeutic agents and delivery systems leave room for improvement. One of the most promising targets for new generation chemotherapeutic agents is the MAPK pathway, and a number of inhibitors against various key proteins in this pathway are being developed.

The main limitations of conventional in vitro models of cancer (cell monolayers) are that they are not representative of cancer heterogeneity and that they do not account for the tumor microenvironment. Both these parameters influence cancer behavior [2-4]. Additionally, cells in monolayers tend to be more sensitive to drugs, since their growth is subject to both tensile [5] and oxidative stress [6]. Inaccuracies at the in vitro and in vivo levels lead to a low rate of translational success in the clinic, despite therapeutics passing initial stages of drug development $[7,8]$.

The use of 3D in vitro cancer models has revolutionized cancer research, allowing a more controlled environment for cell proliferation to be studied in the laboratory. Culturing cells in 3D elicits change in proliferation, behavior and protein expression compared with monolayers [9-11]. In addition, 3D cancer models allow drug penetration factors to be investigated [12,13]. The most common approach for 3D cell culture uses cells grown as spheroids. However promising, the highly cell-dense structure and thickness (in the range of hundreds of microns) of spheroid models tend to promote necrosis in the inner layers, due to the scarcity of nutrients and oxygen in their core [14]. One alternative is the use of natural scaffolds, which mimic
Víctor López-Dávila', Tarig Magdeldin ${ }^{1,2}$, Hazel Welch', Miriam Victoria Dwek ${ }^{3}$, Ijeoma Uchegbu ${ }^{4}$ \& Marilena Loizidou $*, 1$

${ }^{1}$ Cancer Nanotechnology Group, University College London, Division of Surgery \& Interventional Science, Royal Free Campus, London, NW3 2PF, UK ${ }^{2}$ Institute of Orthopaedics \& Musculoskeletal Sciences, University College London, Division of Surgery \& Interventional Science, Stanmore Campus, HA7 4LP, UK ${ }^{3}$ Department of Biomedical Sciences, Faculty of Science \& Technology, University of Westminster, $115 \mathrm{New}$ Cavendish Street, London, W1W 6UW, UK

${ }^{4}$ School of Pharmacy, University College London, 29-39 Brunswick Square, London, WC1N 1AX, UK *Author for correspondence: Fax: +44 2074726444 m.loizidou@ucl.ac.uk 
the tumor microenvironment and contain constituent proteins, such as collagen type I or hyaluronic acid [1517]. While the use of collagen type I hydrogels mimics the extracellular matrix and is a promising approach, its main limitation is the high water content [18]. Here we use a plastic compression technique to create tumoroids, 3D in vitro models of colorectal cancer, by removing the excess water from collagen type I hydrogels. This model mimics the mechanical properties of real tissue better than uncompressed collagen hydrogels $[9,19,20]$.

A number of organic nanoparticles have shown promising results in cancer therapy as delivery platforms for chemotherapeutic drugs, with liposomes and micelles in the vanguard of development [21-24]. These carriers are particularly useful for the delivery of therapeutics with low water solubility [25].

Two of the most promising organic nanocarriers are cationic liposomes made from 3 beta- $\left(\mathrm{N}-\left[\mathrm{N}^{\prime}, \mathrm{N}^{\prime}-\right.\right.$ dimethylaminoethane] carbamoyl) cholesterol (DCchol) and dioleoylphosphatidylethanolamine (DOPE), which have been shown to have potential as nucleic acid and drug nanocarriers [26-28], and micelles made from $\mathrm{N}$-palmitoyl-N-monomethyl-N,N-dimethyl-N,N,Ntrimethyl-6-O-glycolchitosan (GCPQ), also cationic in nature, which have been used to solubilize and orally deliver hydrophobic molecules with high efficiency [29-31]. Cationic nanoparticles have the advantage of improving internalization by interacting with the anionic cell surface $[22,32,33]$. However, only a few of these nanoparticles have been tested in $3 \mathrm{D}$ in vitro cancer models, and most of these were spheroids [34-36].

A number of novel chemotherapeutic agents have been developed over the last years. Two of the most common target proteins for these therapies are MEK1 and MEK2, key proteins of the MAPK pathway, particularly in cancers that have mutations in $R A S$ or $R A F$ since both molecules are upstream of MEK1/2 in the pathway. Here we explore the efficacy of AZD6244, an inhibitor of MEK1/2, in its free form as well as loaded in cationic liposomes and micelles, and we compare the efficacy of different formulations on cell monolayers and tumoroids. We chose the cell line HCT116 for having a mutated $K R A S$ that continuously activates the MAPK pathway. To our knowledge, this is the first time nanoparticles have been tested in a natural scaffold-based 3D in vitro cancer model.

\section{Materials \& methods}

Nanoparticle fabrication

Liposomes were fabricated following a modified version of Mozafari's method [37]. Equimolar concentrations $(5 \mathrm{mM})$ of DOPE and DC-cholesterol (Avanti polar lipids, AL, USA) were dissolved in chloroform- methanol (2:1), together with $2 \mathrm{mM}$ AZD6244 or Nile Red, for drug toxicity and penetrations studies respectively, to a total of $9 \mathrm{ml}$. Solvents were removed by rotaevaporation to obtain a thin hydrophobic layer attached to the glass surface. That layer was rehydrated with $10 \mathrm{ml}$ Milli-Q water, vortexed for 1 minand ultrasonicated for $5 \mathrm{~min}$ until fully detached. The resulting suspension was extruded through a $200 \mathrm{~nm}$ polycarbonate membrane using a liposome extruder (Avanti polar lipids) and dialyzed against Milli-Q water for six 2 -h cycles. Micelles were fabricated following a modified version of the protocol described in [29]. $20 \mathrm{mg}$ of GCPQ polymer (palmitoylation $=18.9 \%$, quaternalization $=11.2 \%, \mathrm{MW}=17,120 \mathrm{~g} / \mathrm{mol}$ ) were dispersed in Milli-Q water either alone, with $2 \mathrm{mg}$ of AZD6244 or with $0.5 \mathrm{mg}$ of Nile Red for control toxicity, drug toxicity and penetration studies, respectively. The mixture was vortexed and sonicated for $1 \mathrm{~min}$ until it became homogeneous and then probesonicated with amplitude 45 for six 5-min cycles alternated with 3-min breaks (Qsonica, CT, USA) in an ice bath. The newly formed GCPQ micelles were allowed to stabilize for $15 \mathrm{~min}$ at room temperature before being filtered through a $0.45 \mu \mathrm{m}$ filter and centrifuged at $2000 \mathrm{x}$ g to remove nonencapsulated drug crystals.

\section{Nanoparticle characterization}

The drug content of both formulations was determined by HPLC against a standard curve of free AZD6244. Samples were diluted in methanol (1:3 for micelles and 1:25 for liposomes) to break the nanoparticles open and then filtered through a $0.2 \mu \mathrm{m}$ membrane prior to the chromatographic analysis. $30 \mu \mathrm{l}$ of the sample were loaded into the loop and separated by reversed-phase chromatography using a Luna $\mathrm{C} 18$ column comprising $5 \mu \mathrm{m}$ beads, $4.6 \mathrm{~mm}$ diameter, $100 \mathrm{~mm}$ length, fitted with a Safeguard precolumn (Phenomenex, Cheshire, UK). The assays were performed at $25^{\circ} \mathrm{C}$, using a TM717 autosampler, a TM515 HPLC pump and a TM486 absorbance detector set at a wavelength of 260 nm (Waters, Hertfordshire, UK). The mobile phase was HPLC grade methanol with an isocratic flow rate of $0.8 \mathrm{ml} / \mathrm{min}$, and AZD6244 showed a retention time of 21.5 min under these conditions.

Dynamic light scattering (DLS) was used to determine the size of nanoparticle aggregates in an aqueous solution. A diluted suspension of nanoparticles was placed in a disposable cuvette and measured threetimes for 15 repeats each for size analysis in a Malvern Nanosizer ZS (Malvern, Worcestershire, UK).

Transmission electron microscopy (TEM) was used to visualize the particles at the nanoscale, and to determine their morphology and approximate size. An aqueous suspension of nanoparticles was placed on a 
carbon grid and stained with a $1 \% \mathrm{w} / \mathrm{v}$ aqueous solution of uranyl acetate. TEM images were taken at a minimum of $30000 \mathrm{X}$ magnification using a Philips CM120 BioTwin transmission electron microscope (Philips, Eindhoven, The Netherlands) with a AMT 5 megapixel digital camera (AMT, UK).

\section{Cell maintenance}

HCT116 human colorectal carcinoma cells were obtained from the European Collection for Cell Cultures (Sigma-Aldrich, Dorset, UK) and routinely cultured in McCoy's 5A medium supplemented with $10 \% \mathrm{v} / \mathrm{v}$ foetal bovine serum, $100 \mathrm{U} / \mathrm{ml}$ penicillin and $100 \mu \mathrm{g} / \mathrm{ml}$ streptomycin (P/S) (Invitrogen, Paisley, $\mathrm{UK}$ ), at $37^{\circ} \mathrm{C}$ and in a $5 \% \mathrm{CO}_{2} /$ air humidified atmosphere.

\section{Monolayer \& tumoroid cell culture}

For toxicity experiments in cell monolayers, 30,000 cells/well were seeded in 24-well plates, fed with $500 \mu \mathrm{l}$ of McCoy's $5 \mathrm{~A}$ medium supplemented with fetal bovine serum (FBS) and P/S and allowed to grow for $24 \mathrm{~h}$ before substituting the culture medium with serumfree medium. Serum-free conditions minimize interactions, such as agglomeration, between the free drug or nanoformulations with serum proteins; cell cycles were also semisynchronized. For toxicity and penetration experiments in 3D, tumoroids were fabricated using the plastic compression method modified from [38]. Briefly, $400 \mu \mathrm{l}$ of MEM x10 medium were mixed with $3.2 \mathrm{ml}$ of rat tail collagen type I $(2.20 \mathrm{mg} / \mathrm{ml}$ in $0.6 \% \mathrm{v} / \mathrm{v}$ acetic acid; First Link UK Ltd). The mixture was neutralized with $5 \mathrm{M}$ and $1 \mathrm{M} \mathrm{NaOH}$ until a color change from yellow to pink was observed, and then $6.4 \times 10^{6}$ cells suspended in $400 \mu \mathrm{l}$ medium were added to the mixture. The resulting solution was placed in a stainless steel mould and left to gel for $30 \mathrm{~min}$ at room temperature. The mould containing the gel was then placed on a nylon mesh resting on a $165 \mu \mathrm{m}$ stainless steel mesh placed on a $30 \mathrm{~nm}$ filter paper. A $176 \mathrm{~g}$ plunger was placed on top of the gel allowing compression for $30 \mathrm{~s}$ and the whole compression process was repeated on the other side of the gel. The resulting $2.6 \% \mathrm{w} / \mathrm{v}$ collagen dense mass [20] was cut in four equal parts with a surgical scalpel and nested in $1 \mathrm{ml}$ of uncompressed acellular collagen hydrogel in 12-well plates. The nested construct was left for $20 \mathrm{~min}$ to gel at $37^{\circ} \mathrm{C}$. Fully supplemented medium $(1 \mathrm{ml})$ was added; cells were starved with serum-free media on day 6 and treated with the therapeutic formulations on day 7.

For Western blot analysis, $2 \times 10^{5}$ HCT116 cells were seeded on 6 -well plates and left to grow until $60 \%$ confluence. At that point, cells were treated with a range of AZD6244 concentrations $(0.01-1 \mu \mathrm{M})$ either free or in nanoparticle formulations for $1 \mathrm{~h}$ and then $10 \mathrm{ng} / \mathrm{ml}$ of EGF were added for 10 min to activate the MAPK pathway. Cells were then lysed with $250 \mu \mathrm{l}$ of radioimmunoprecipitation assay buffer (Sigma-Aldrich) supplemented with protease inhibitors (Roche, Hertfordshire, UK) and samples were stored at $-20^{\circ} \mathrm{C}$ until required.

\section{Toxicity experiments}

For toxicity experiments on cell monolayers, after $24 \mathrm{~h}$ of starvation (in serum-free media), cells were treated with a range of AZD6244 concentrations $(0.01-10 \mu \mathrm{M})$ in free or nanoparticle form, or the equivalent in the form of control nanoparticles. Free drug and nanoformulation concentrations were prepared in serum-free McCoy's 5A medium. After $48 \mathrm{~h}$ treatment, metabolic activity and cell proliferation were determined using MTT and total DNA assays, respectively. For the MTT assay, $12.5 \mu \mathrm{l}$ of a $20 \mathrm{mg} / \mathrm{ml}$ MTT solution (SigmaAldrich) was added to each well and left for $30 \mathrm{~min}$ before removing the medium, dissolving the resulting crystals in $400 \mu \mathrm{l}$ of DMSO, incubating for $10 \mathrm{~min}$ at $37^{\circ} \mathrm{C}$ and reading the absorbance at $570 \mathrm{~nm}$ in a colorimetric plate reader (Anthos 2010 Standard, Biochrom, Cambridge, UK). For the total DNA assay, the Hoechst stain total DNA kit (Sigma-Aldrich) was used. Medium was removed from each well, $500 \mu \mathrm{l}$ of DNAse-free water added and three freeze-thaw cycles were performed. $100 \mu \mathrm{l}$ of the resulting solution were added to $100 \mu \mathrm{l}$ of a 1:1000 dilution of Hoechst 33298 dye solution, fluorescence was read immediately after and total DNA extrapolated from a standard curve (excitation = $350 \mathrm{~nm}$, emission $=460 \mathrm{~nm}$ ) in a Fluoroskan Ascent FL (Thermo Labsystems, Warrington, UK).

For toxicity experiments on tumoroids, cells were starved on day 6 and treated on day 7 with the same range of AZD6244 concentrations than the monolayers. Alamar Blue ${ }^{\circledR}$ assay (Invitrogen, Paisley, UK) was used to measure metabolic activity. Concentrated Alamar Blue was diluted at a 1:10 ratio in Phenol Redfree DMEM medium. Medium was removed from wells containing the nested constructs and $1 \mathrm{ml}$ of the diluted Alamar Blue ${ }^{\circledR}$ solution was added. Tumoroids were then incubated for $4 \mathrm{~h}$ in a $37^{\circ} \mathrm{C}, 5 \% \mathrm{CO}_{2} /$ air humidified atmosphere, $100 \mu \mathrm{l}$ of the supernatant was transferred to a black 96 -well plate and the fluorescence read immediately (excitation $=530 \mathrm{~nm}$, emission $=620$ $\mathrm{nm}$ ) in a Fluoroskan Ascent FL (Thermo Labsystems).

\section{Protein analysis}

Protein samples extracted using radioimmunoprecipitation assay buffer were centrifuged at $12000 \mathrm{x} \mathrm{g}$ at $4^{\circ} \mathrm{C}$ for $5 \mathrm{~min}$ to remove cell debris and total protein was quantified using the Lowry protein assay (Ther- 
moscientific, Warrington, UK), at reading absorbance $750 \mathrm{~nm}$ using a Jenway Genova Plus Spectrophotometer (Jenway, Staffordshire, UK). After mixing with NuPAGE ${ }^{\circledR}$ LDS Sample Buffer (Life technologies) containing $12.5 \% \beta$-Mercaptoethanol (SigmaAldrich) protein samples were denatured at $95^{\circ} \mathrm{C}$ for 5 min, chilled on ice, then loaded in a NuPAGE $4-12 \%$ Bis-Tris polyacrylamide gel (Invitrogen) next to a prestained protein ladder (SeeBlue ${ }^{\circledR}$ Prestained, Invitrogen). The gel was run at $150 \mathrm{~V}$ for $90 \mathrm{~min}$ in MES SDS running buffer, and transferred to PVDF membranes (Immun-Blot ${ }^{\circledR}$ PVDF membrane, BioRad, Hertfordshire, UK) by immersing the cassette in NuPAGE ${ }^{\circledR}$ Transfer Buffer (Life Technologies) and running it at $30 \mathrm{~V}$ for $75 \mathrm{~min}$. Membranes were blocked with $2.5 \% \mathrm{w} / \mathrm{v}$ bovine serum albumin (BSA) in PBS-Tween solution and incubated with mouse monoclonal anti-pERK (1:1000 dilution) (\#9106S, Cell signaling, Hitchin, UK) and GAPDH (1:2000 dilution) (\#sc-32233, Santa Cruz Biotechnology, CA, USA) antibodies in $1 \%$ BSA, followed by hybridization using a horseradish peroxidase (HRP)-conjugated goat antimouse secondary antibody (1:2000 dilution) (\#sc-2005, Santa Cruz Biotechnology), developed with Bio-Rad Immuno-Star Western C developer and visualized using a BioRad Molecular Imager ${ }^{\circledR}$, ChemiDoc $^{\mathrm{TM}}$ XRS+ with ImageLab ${ }^{\mathrm{TM}}$ Software (BioRad).

\section{Penetration studies}

Nile red liposomes and micelles were fabricated as indicated above. The fluorescent signal of the solution was normalized for all formulations (liposomes, micelles and free dye dissolved in DMSO). Confocal microscopy was used to focus on an optical plane $300 \mu \mathrm{m}$ deep from the top of the artificial cancer mass (ACM) (approximately its core), and images were taken using a green laser $(543 \mathrm{~nm})$ and a red fluorescence detector $(630 \mathrm{~nm})$. Culture medium was removed and the Nile red formulations were added on the surface of the tumoroids. Images of the central plane inside the compressed cancer mass were taken over a period of $24 \mathrm{~h}$ to determine the rate of penetration of each formulation. Fluorescent counts were quantified using Image J.

\section{Statistical analysis}

Statistical analysis and graphical representations were performed using the GraphPad Prism5.0 software. Data were analyzed using Student's $t$-test and one-way ANOVA analysis followed by Dunnet's or Bonferronni's post hoc tests for statistical significance. Means and standard deviations are represented for each treatment group and all statistical analyses were carried out on the original data.

\section{Results}

DOPE/DC-cholesterol \& GCPQ nanoparticles encapsulating AZD6244

DOPE/DC-cholesterol liposomes were fabricated by dissolving the lipid components and hydrophobic cargos (AZD6244, Nile red or none) in a mixture of chloroform and methanol (2:1) and evaporating the solvents to rehydrate the remaining thin layer. The type of cargo did not influence the size or morphology of the liposomes, which remained spherical and in the same size range, as imaged by TEM (Figure 1A). Liposome sizes ranged between $70 \mathrm{~nm}$ and $500 \mathrm{~nm}$ with the majority at approximately $165 \mathrm{~nm}$, as shown by DLS analysis (Figure 2A). Occasionally, DLS analysis also revealed a very small peak at 3-6 $\mu \mathrm{m}$, indicating the tendency of these nanoparticles to form aggregates in aqueous media. In the case of the control liposomes with no cargo, the main peak at $180 \mathrm{~nm}$ was very similar to that observed for the cargo-loaded nanoparticles, but with a broader size range $(30-1100 \mathrm{~nm}$ ) (data not shown).

GCPQ micelles were fabricated by tip sonication of an aqueous solution containing GCPQ and the hydrophobic cargo, or just GCPQ for the control nanoparticles. After an additional centrifugation and filtration step to remove nonencapsulated drug, TEM analysis revealed a spherical morphology for micelles, but these were smaller than the liposomes (Figure 1B); as in the case of the liposomes, micelle size was not influenced by the type of cargo. As shown by TEM, micelle diameters were on average approximately $30 \mathrm{~nm}$ in diameter while DLS analysis shows a distinct peak at $350 \mathrm{~nm}$, covering from 260 to $550 \mathrm{~nm}$ (Figure 2B). This is probably due to the tendency of GCPQ micelles to form aggregates in aqueous media [29]. By contrast, control micellar nanoparticles with no cargo formed two different sized aggregates in solution, showing peaks at 9 and $50 \mathrm{~nm}$ in DLS with ranges of 5-12 $\mathrm{nm}$ and $40-80$ $n m$ respectively (data not shown).

As measured by HPLC, both drug-loaded nanoparticles yielded AZD6244 concentrations between 0.85 and $1.15 \mathrm{mM}$, representing encapsulation efficiencies between 45 and 55\%.

\section{Nanoformulations increase AZD6244 efficacy in monolayers}

Human colorectal cancer cells (HCT116) were treated with micelles and liposomes containing AZD6244 as well as free AZD6244 for $48 \mathrm{~h}$ before the total DNA and MTT assays were performed. In terms of cellular proliferation (determined by total DNA assay) AZD6244 treatment resulted in a $21 \%$ decrease in proliferation at the highest concentration of the drug $(1 \mu \mathrm{M})$, while liposomes and micelles with the same drug load resulted 


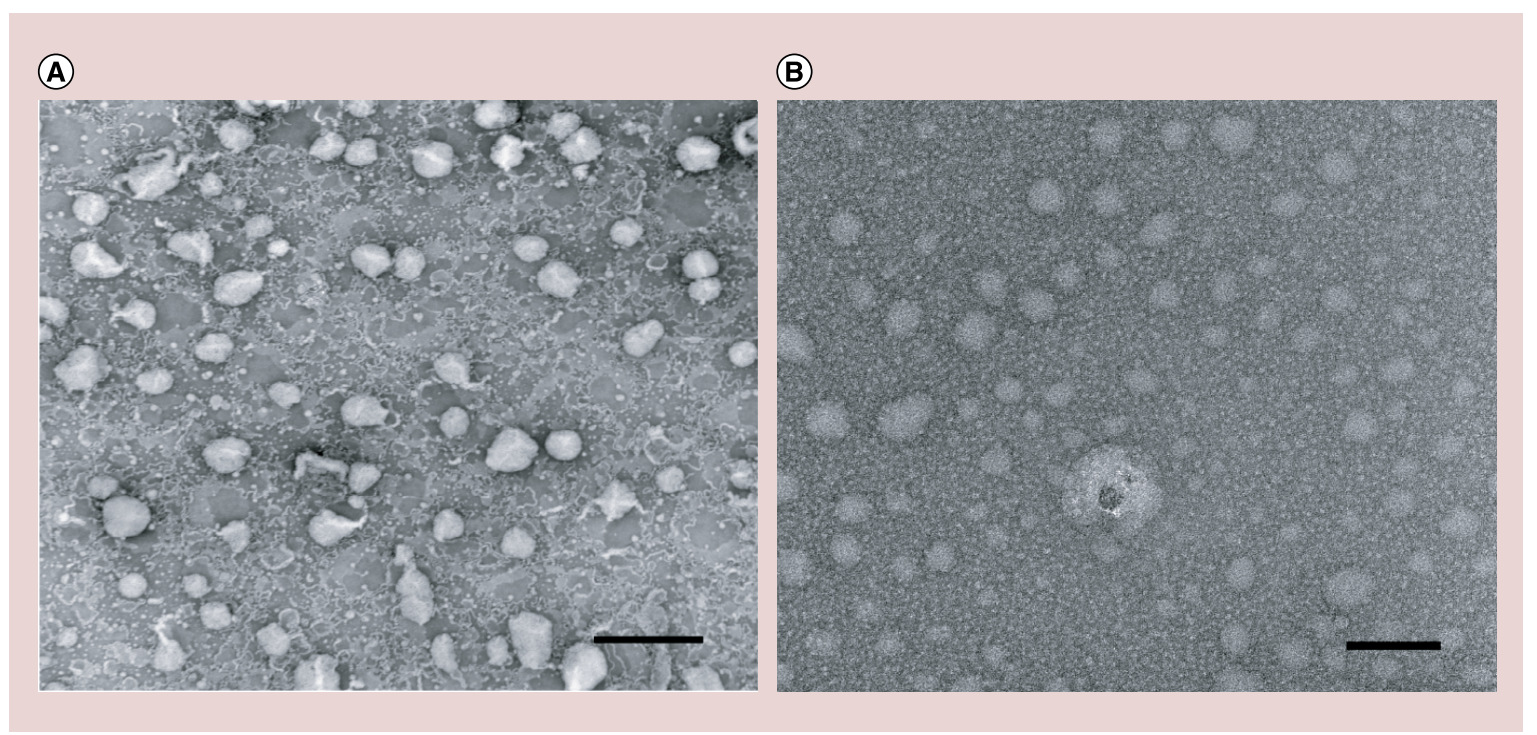

Figure 1. Transmission electron microscopy images of organic nanoparticles. Aqueous suspensions of AZD6244loaded nanoparticles were placed on a carbon grid and stained with a $1 \%$ aqueous solution of uranyl acetate. (A) DOPE/DC-cholesterol liposomes (scale bar: $500 \mathrm{~nm}$, mag 31000x, 80.0 V). (B) GCPQ micelles (scale bar: $100 \mathrm{~nm}$, mag $135000 \times$, HV120.0 kV).

in 61 and $89 \%$ inhibition, respectively (Figure 3A; $\mathrm{p}<0.001$ ). Regarding metabolic activity measured by MTT, nanoformulations were clearly more efficient than the free drug but the differences were not as pronounced (18, 60 and 40\% for the free drug, liposomes and micelles, respectively) (Figure 3B; $\mathrm{p}<0.001$ ). This may be due to an increase in metabolic activity by the surviving cells which need to compete less for the nutrients in the medium. There was a statistically significant difference between micelle and liposomal formulations at every concentration tested, except at $0.01 \mu \mathrm{M}$ using the proliferation assay and at $0.1 \mu \mathrm{M}$ when using the MTT assay. Interestingly, micelles appeared more toxic according to the total DNA assay, while MTT assay revealed a higher metabolic activity inhibition in the liposometreated group, suggesting differences in the delivery and internal processing between these nanoformulations.

To establish whether the above toxicity patterns were due to more efficient delivery of AZD6244 into the cell, internal processing or merely due to the toxicity of the nanoparticle components, a similar experiment with water-containing nanoparticles was performed. The highest concentration of nanoparticles (equivalent to the concentration used for the $1 \mu \mathrm{M}$ AZD6244 treatment) elicited 7 and $25 \%$ proliferation inhibition for liposomes and micelles, respectively (Figure 3C). This suggests that most of the inhibitory effect of the nanoformulations is due to a more efficient delivery and internal processing of the drug and not due to an intrinsic toxicity of the nanoparticle components.

To further confirm delivery of AZD6244 into the cytoplasm and specific pharmacological action on the target MAPK pathway, Western Blot analysis was performed. Cells were treated with the same drug concentrations in the three different delivery forms and proteins were separated by size in a polyacrylamide gel. Phosphorylated ERK, a common indicator of MEK activity, was detected using a monoclonal antibody. Densitometry revealed that in all three formulations, a dose-dependent reduction in the levels of phosphorylated ERK occurred; these decreases were 86, 80 and $53 \%$ for liposomes, micelles and free drug, respectively (Figure 4). This shows that AZD6244 reaches its target and elicits a specific response at the protein level, and that the delivery is much more efficient in nanoformulations compared with the free drug. While control GCPQ micelles are significantly more toxic than control DOPE/DC-cholesterol liposomes (Figure 3C), AZD6244-loaded liposomes elicit more inhibition of metabolic activity and MEK1/2 activity than AZD6244-loaded micelles (Figures 3B \& 4), suggesting a more efficient delivery of the drug.

\section{GCPQ micelles elicit the highest toxicity in a 3D in vitro cancer model}

The 3D in vitro cancer model used here consisted of a dense collagen core containing the cancer cells (ACM) surrounded by an uncompressed collagen hydrogel (stromal surround) (Nyga et al. 2013). Tumoroids were allowed to mature for 7 days, allowing cancer cells to form aggregates, thereby mimicking in vivo avascular micrometastases. Treatment with free AZD6244 and the nanoformulations was performed for 24, 48 and $72 \mathrm{~h}$. It is routine to include an extra $24 \mathrm{~h}$ incubation 
(A) Size distribution by intensity

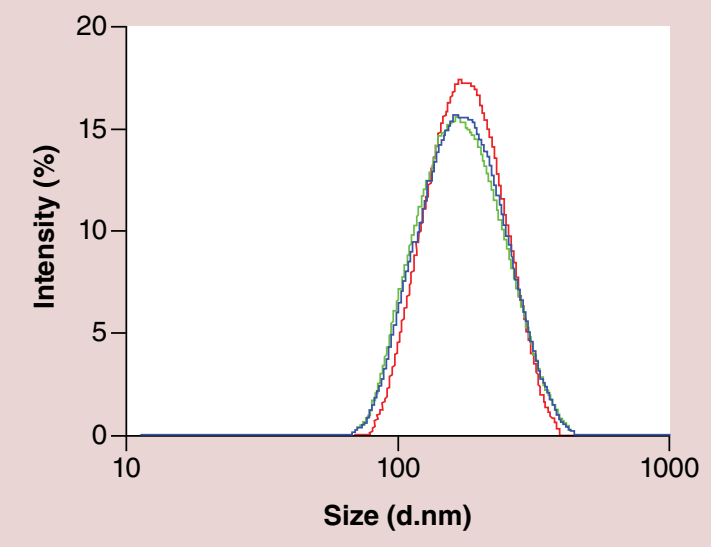

(B) Size distribution by intensity

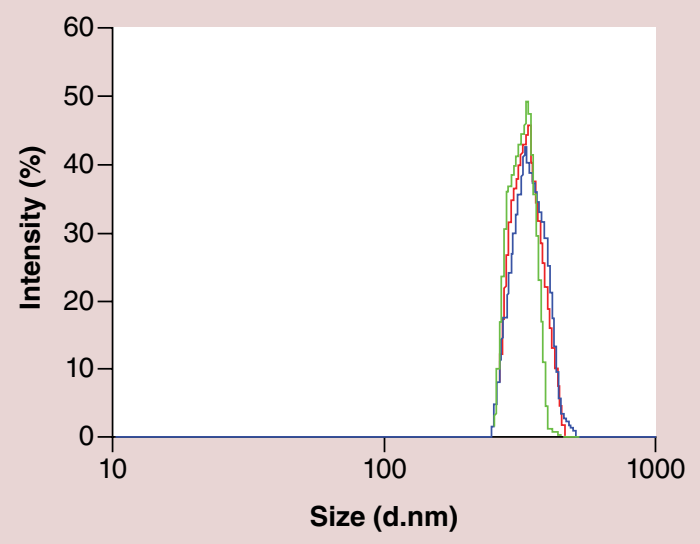

Figure 2. Dynamic light scattering analysis of organic nanoparticles. Aqueous suspensions of AZD6244loaded nanoparticles were placed in a disposable cuvette and measured three-times (each in one color) for 15 repeats each for size analysis in a Malvern Nanosizer ZS. (A) AZD6244-loaded DOPE/DC-cholesterol liposomes. (B) AZD6244-loaded GCPQ micelles.

when moving from $2 \mathrm{D}$ to $3 \mathrm{D}$ cultures to optimize the effects of drugs, as more time is needed for the drug to reach the cells in 3D and the metabolism in 3D tumoroids is also slower (based on data generated from our group, not shown). For that reason, when correlating the data between both models, the $48 \mathrm{~h}$ treatment in $2 \mathrm{D}$ was compared with the $72 \mathrm{~h}$ treatment in $3 \mathrm{D}$. Treatment with free AZD6244 and its nanoformulations showed increasing efficacy with increasing AZD6244 concentrations and treatment times. Inhibition of metabolic activity started to be significant at $0.1 \mu \mathrm{M}$.

Drug efficacy in tumoroids was different from monolayers. When comparing the results of metabolic activity assays, while the free drug was more effective in $3 \mathrm{D}$ than in $2 \mathrm{D}$ (38 vs $18 \%$ inhibition), the nanoparticle treatments were more effective in $2 \mathrm{D}(60$ vs $26 \%$ inhibition for liposomes and 40 vs $34 \%$ inhibition for micelles) (Figure 5C). Overall, micelles were more toxic in tumoroids than the other formulations (Figure 5). Significant differences were observed at 0.1 $\mu \mathrm{M}$ AZD6244 and at $24 \mathrm{~h}$, where micelles were superior to liposomes (Figure 5A: $\mathrm{p}<0.01$ ). Furthermore, at $1 \mu \mathrm{M}$ and $48 \mathrm{~h}$, micelles were superior to both liposomes $(\mathrm{p}<0.001)$ and free drug $(\mathrm{p}<0.01)$ (Figure $5 \mathrm{~B})$, while at $1 \mu \mathrm{M}$ and $72 \mathrm{~h}$ only micelles were only superior to liposomes $(\mathrm{p}<0.05)$. In the latter treatment, the free drug also showed more toxicity than the liposomes $(\mathrm{p}<0.01)$, making the liposomes the least effective of the three formulations in tumoroids (Figure $5 \mathrm{C}$ ).

Nanoparticle-mediated delivery is hindered by poor penetration into the ACM

In order to further explore the differences in the efficacy of nanoparticles for delivery of drug in the two models, tumoroids were cultured and matured for 7 days and exposed to Nile red-loaded fluorescent nanoparticles. The focal plane was set at $300 \mu \mathrm{m}$ into the ACM core, and fluorescence was imaged and measured over time.

These studies revealed a clear size-dependent ability of the nanoparticles to penetrate the ACM. The penetration of the free dye control at the ACM core started reaching a plateau before $2 \mathrm{~h}$ (Figure 6A \& C), with a fluorescent signal 10-fold higher than the one showed by liposomes and micelles after $24 \mathrm{~h}$ (Figure 6B \& C). GPCQ micelles and DOPE /DC-cholesterol liposomes showed a very similar penetration profile, most likely due to the fact that they are both cationic.

\section{Discussion}

This work explores the effect of cationic organic nanocarriers loaded with AZD6244 on HCT116 colorectal cancer cells cultured both as monolayers and in tumoroids. The use of $3 \mathrm{D}$ cancer models aims to mimic the cell microenvironment found in vivo while still allowing the control of parameters in vitro. AZD6244 has been extensively studied in Phase I and II clinical trials with promising results, alone and in combination with other therapies [39].

\section{Nanoparticle toxicity \& AZD6244 delivery}

To our knowledge, this is the first report on AZD6244carrying nanoparticles, although other MEK inhibitors have been encapsulated in nanoformulations. Basu et al. demonstrated that PLGA nanoparticles carrying the MEK inhibitor PD98059 successfully induced apoptosis and inhibited proliferation of lung cancer and melanoma in vitro, and improved the effect of cisplatin in melanoma cells in vivo [40]. Similarly, 
a DGL cationic liposome containing a combination of the MEK inhibitor PD0325901 and Mcl I-specific siRNA reduced KB cells survival in vitro and induced $79 \%$ apoptosis in vivo [41].

Our results demonstrated that DOPE/DC-cholesterol cationic liposomes exert very little toxicity in the absence of AZD6244 (7\% inhibition at the highest concentration, Figure $3 \mathrm{C}$ ), which in comparison to the AZD6244-loaded liposomes (61\% for total DNA and $60 \%$ for metabolic activity, Figure $3 A$ \& B) suggests a very successful AZD6244 delivery mechanism. On the other hand, reports regarding the effect of cationic surfaces on cells could explain the toxicity of GCPQ nanoparticles in the absence of AZD6244 (25\% inhibition at the highest concentration, Figure $3 \mathrm{C}$ ), which were significantly more toxic than the liposomes ( $\mathrm{p}<0.001$, Figure $3 \mathrm{C}$ ), but not nearly as toxic as the drug-loaded GCPQ micelles ( $40 \%$ for metabolic activity and $89 \%$ for total DNA, Figure $3 A$ \& B).

Aside from the higher toxicity of GCPQ micelles compared with DOPE/DC-cholesterol liposomes, both AZD6244-loaded nanoparticles seemed to be excellent delivery platforms as determined by proliferation (total DNA assay), metabolic activity (MTT assay) and enzyme inhibition (pERK levels). The toxicity of both control nanoparticles was significantly lower than that of the AZD6244-loaded nanoparticles (Figure 3). As determined by Western blot analysis of phosphorylated ERK1/2, all three formulations successfully inhibited the activity of MEK1/2 (Figure 4). The most efficient inhibition was elicited by the liposomal formulation. These results, in combination with the much higher cytotoxicity of liposomes and micelles compared with the free drug $(61,89$ and $21 \%$ inhibition of proliferation, and 60, 40 and $18 \%$ inhibition in metabolic activity, respectively [Figure 3]) suggest an efficient internal processing and delivery to the cytoplasm. Specifically, liposomes seem to be the best route of delivery in monolayers, since they inhibit metabolic activity (Figure 3B) and MEK activity (Figure 4) rather more efficiently than both micelles and free drug, even though GCPQ micelles show higher toxicity (total
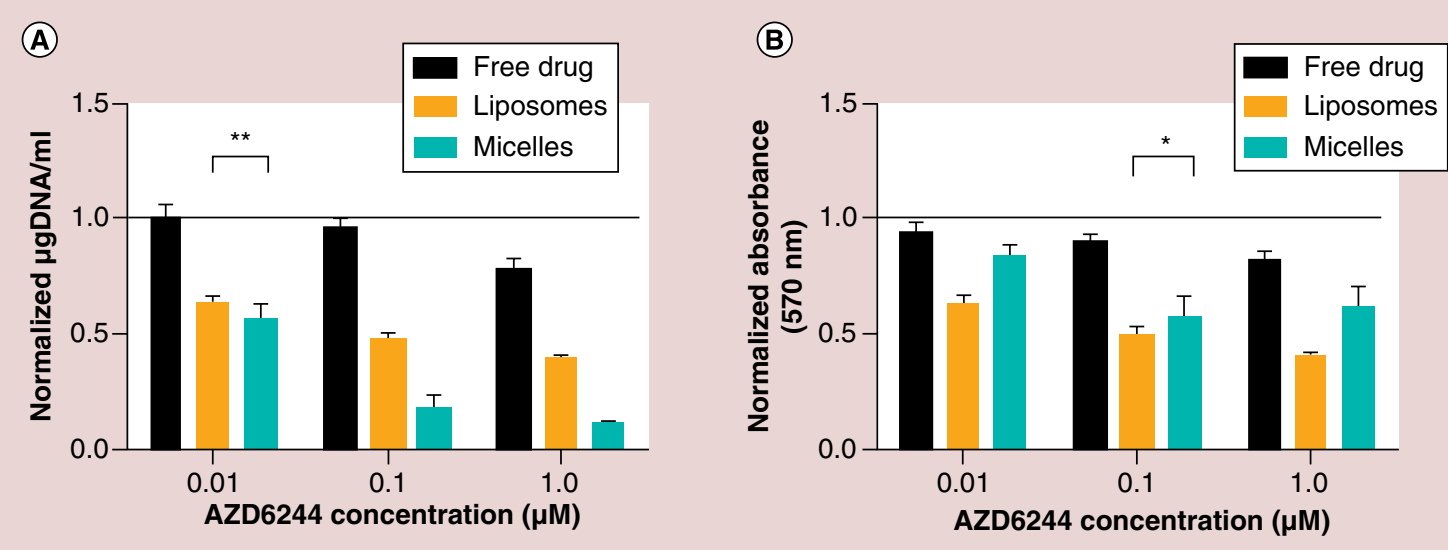

(C)

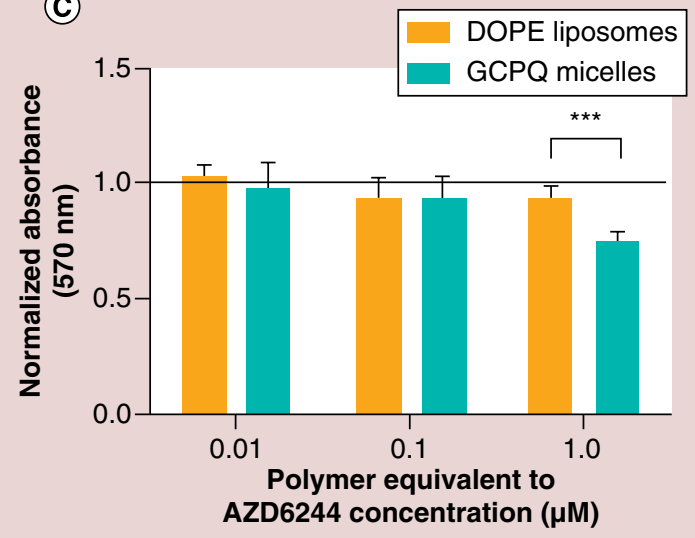

Figure 3. Nanoparticle and free drug toxicity in a 2D in vitro model. HCT116 cells were seeded and maintained in serum-free medium for $24 \mathrm{~h}$ before treatment with free AZD6244 and AZD6244-loaded nanoparticles (A \& B) or control nanoparticles (C). After a 48-h treatment, total DNA assay (A) and MTT assay (B \& C) were performed. Significance: (A) all $p<0.001$ except ** $p<0.01$; (B) all $p<0.001$ except *no significance; (C) ${ }^{* * *} p<0.001$. Control: Thin horizontal line. 
DNA for drug-loaded micelles and MTT for control micelles) (Figure 3A \& C).

Both DOPE/DC-cholesterol liposomes and GCPQ micelles have a positively charged surface. The cationic nature of these nanoparticles not only facilitates nucleic acid loading (as mentioned earlier for the liposomes) but also facilitates the adhesion to the negatively charged cell membrane (through an electrostatic interaction) and internalization [22,32,33], leading to improved delivery and therapeutic effects. The drawback, however, is that a nanoparticle with a surface too rich in positive charges can disrupt the membrane [42]. The presence of DOPE in this cationic liposomes could have the dual function of reducing their toxicity [28] and potentially eliciting an inverted hexagonal phase transition that facilitates the delivery to the cytoplasm [43]. Aside from their cationic nature, there have been reports of liposomal toxicities that may be important in the context of their efficacy. For example, hybrid liposomes with no cargo have been reported to prolong survival in mice with CRC xenografts by inducing apoptosis [44]. The adhesion of liposomes also causes mechanical stress and local contraction of the cell membrane [45]. Compared with these studies, the liposomes used in our work in the absence of AZD6244 showed relatively little toxicity.

However, as a polycation, GCPQ does have a stronger positive surface charge that might destabilize the cell membrane to a certain extent and cause cytotoxicity even in the absence of the drug. While AZD6244-loaded liposomes elicited more proliferation inhibition than AZD6244-loaded micelles in terms of metabolic activity, AZD6244-loaded micelles were more efficient according to the total DNA assay ( $89 \%$ vs $61 \%$, Figure $3 A$ ). This suggests that genotoxicity could be an indirect cause of the inhibition of cellular proliferation in this system. In a study by Shah et al. the effect of a number of nanoparticles with different characteristics on DNA was evaluated: neutral and anionic nanoparticles did not exhibit genotoxic properties while cationic nanoparticles did, as demonstrated by the formation of micronuclei. The degree of genotoxicity did not correlate to the size or molecular weight of the nanoparticles, rather to the extent of the positive charges on the surface of the nanoparticles [46]. This results support the findings reported here of greater nanoparticle toxicity elicited by a polycation such as GCPQ. It is worth noting that in the same work, the overall cytotoxicity of neutral $20 \mathrm{~nm}$ diameter micelles was higher than that of 180 $\mathrm{nm}$ diameter cationic liposomes, indicating that the toxicity of our $30 \mathrm{~nm}$ diameter GCPQ micelles compared with the $165 \mathrm{~nm}$ diameter DOPE/DC-cholesterol liposomes even in the absence of AZD6244 could relate to their size.

According to Andar et al. nanoparticle size affects the way they are internalized. In their work, larger nanoparticles (162.1-nm diameter) tended to be internalized by clathrin-dependent mechanisms while the smallest (40.6-nm diameter) were internalized by dynamin-dependent mechanisms [47]. Smallest particles were internalized 12-fold more efficiently than the largest, and the internalization of intermediatesized nanoparticles corresponded with a size-dependent pattern. Even though this study was carried out with liposomes exclusively, the differences in size apply to our DOPE/DCcholesterol liposomes and GCPQ micelles.

A fraction of our control micelles (without AZD6244) were smaller compared with the AZD6244loaded micelles ( 9 vs $30 \mathrm{~nm}$ ). This could partly explain their toxicity in the absence of AZD6244, since smaller

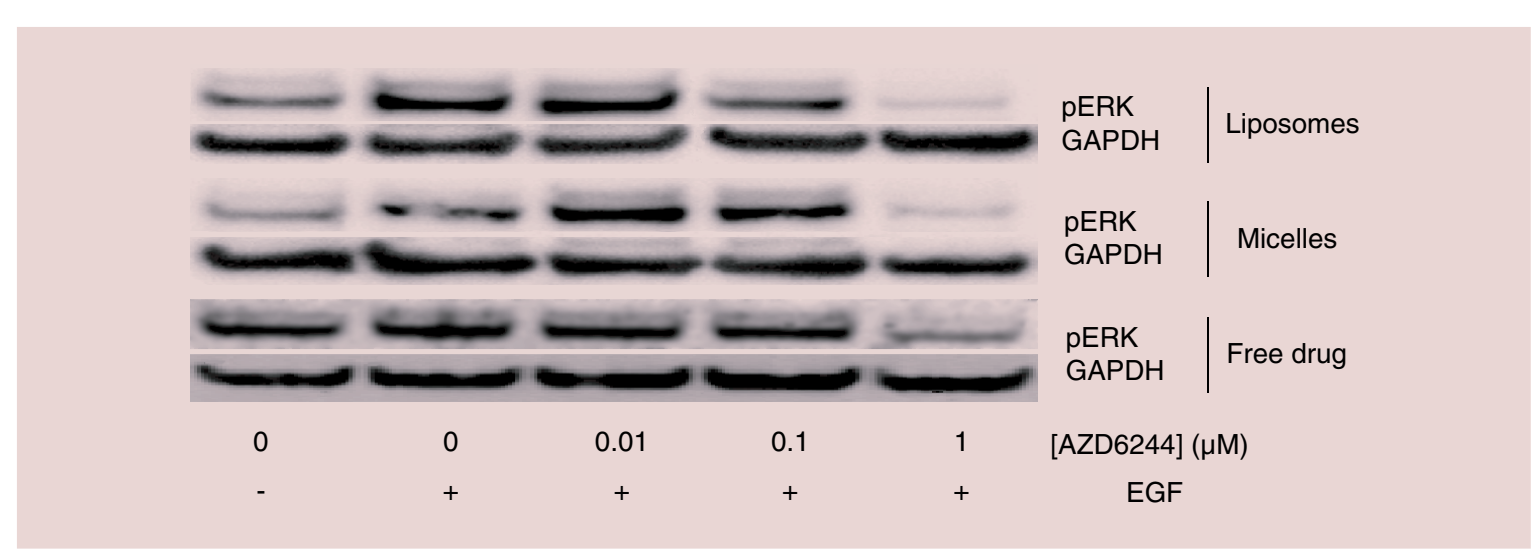

Figure 4. Western blot analysis of phosphorylated ERK. HCT116 cells were seeded and starved for $24 \mathrm{~h}$ before being treated with free AZD6244 and AZD6244-loaded nanoparticles at a range of concentrations for $60 \mathrm{~min}$ followed by the activation of the MAPK pathway by adding $10 \mathrm{ng} / \mathrm{ml} \mathrm{EGF}$ for $10 \mathrm{~min}$. Cells were lysed, protein extracted, quantified and separated using a polyacrylamide gel, transferred to a PVDF membrane and phosphorylated ERK and housekeeping gene GAPDH detected with monoclonal antibodies. 


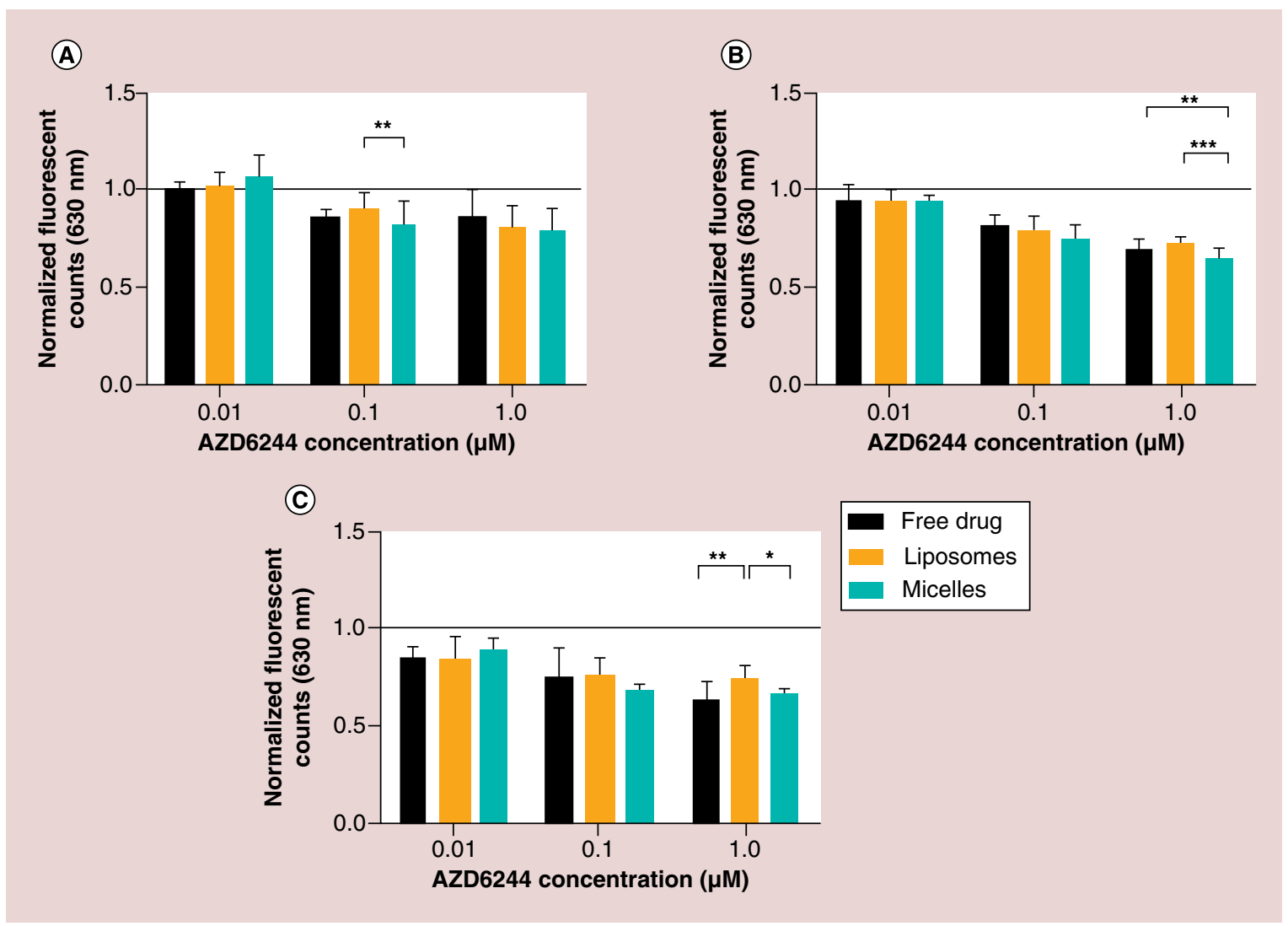

Figure 5. Nanoparticles and free drug toxicity in a collagen-based 3D in vitro model. Cells were seeded in hydrogel-nested artificial cancer masses and left to mature and form aggregates for 7 days. Cells were treated with AZD6244 and AZD6244-loaded nanoparticles at a range of concentrations for 24 (A), 48 (B) and $72 \mathrm{~h}$ (C) before Alamar blue ${ }^{\circledR}$ assay was performed.

${ }^{*} \mathrm{p}<0.05 ; * * \mathrm{p}<0.01 ;{ }^{*} \mathrm{p}<0.001$.

Control: Thin horizontal line.

nanoparticles are more easily internalized and can even reach the cell nucleus [13]. This is supported by the size-dependent cytotoxicity reported by Shah $e t$ al. mentioned earlier [46]. However, since $30 \mathrm{~nm}$ diameter GCPQ micelles were small enough to undergo efficient internalization [47], the toxicity of GCPQ control micelles is likely to be due to their positively charged surface.

\section{Tumoroids as a platform for screening nanoformulations}

A number of MEK inhibitors have been tested in 3D cancer models. These include PD98059 for breast cancer cells using Matrigel [48] and GSK1120212 in combination with BRAF and AURKA inhibitors for melanoma using a skin reconstruction model [49]. As a free drug, the efficacy of AZD6244 has been previously tested in a model of melanoma grown within a collagen hydrogel, in combination with the SCR inhibitor saracatinib showing promising results [50]. This work differs from our plastic compression approach in that cells are cultured in an uncompressed collagen matrix, while our model undergoes plastic compression resulting in a cancer mass with a collagen density of $2.6 \% \mathrm{w} / \mathrm{v}$.

In the present work, treatment with free AZD6244 was more effective in tumoroids than in the monolayers (18\% inhibition at $48 \mathrm{~h}, 1 \mu \mathrm{M}$ in monolayers (Figure 3); 38\% inhibition at $72 \mathrm{~h}, 1 \mu \mathrm{M}$ in tumoroids [Figure 5]). This was especially surprising considering that the cell density in tumoroids is extremely high compared with monolayers, resulting in a drugto-cell ratio over 5000-times higher in the monolayer experiments, and therefore suggesting very different cell behavior between the two models. However, this interpretation must be considered as a hypothesis, since the differences are subtle and the time of treatment differs between both models.

Previous work with spheroids and other 3D models of cancer have shown significant differences in drug response compared with monolayer cultures. However in most cases, 3D cultures tend to be more resistant to antimitotic drugs than monolayers [11,51]. These differences are not properly understood, but cells in 


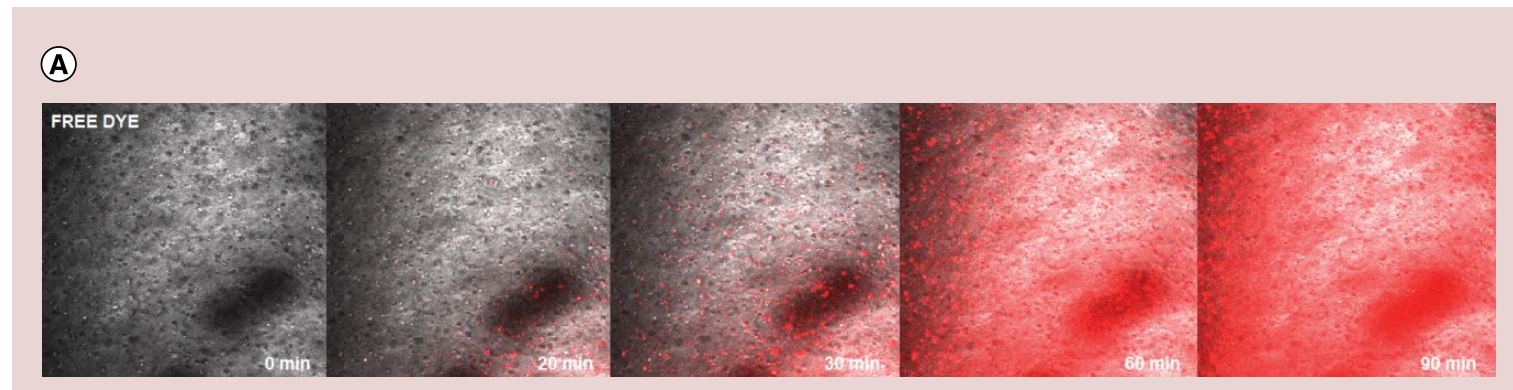

(B)
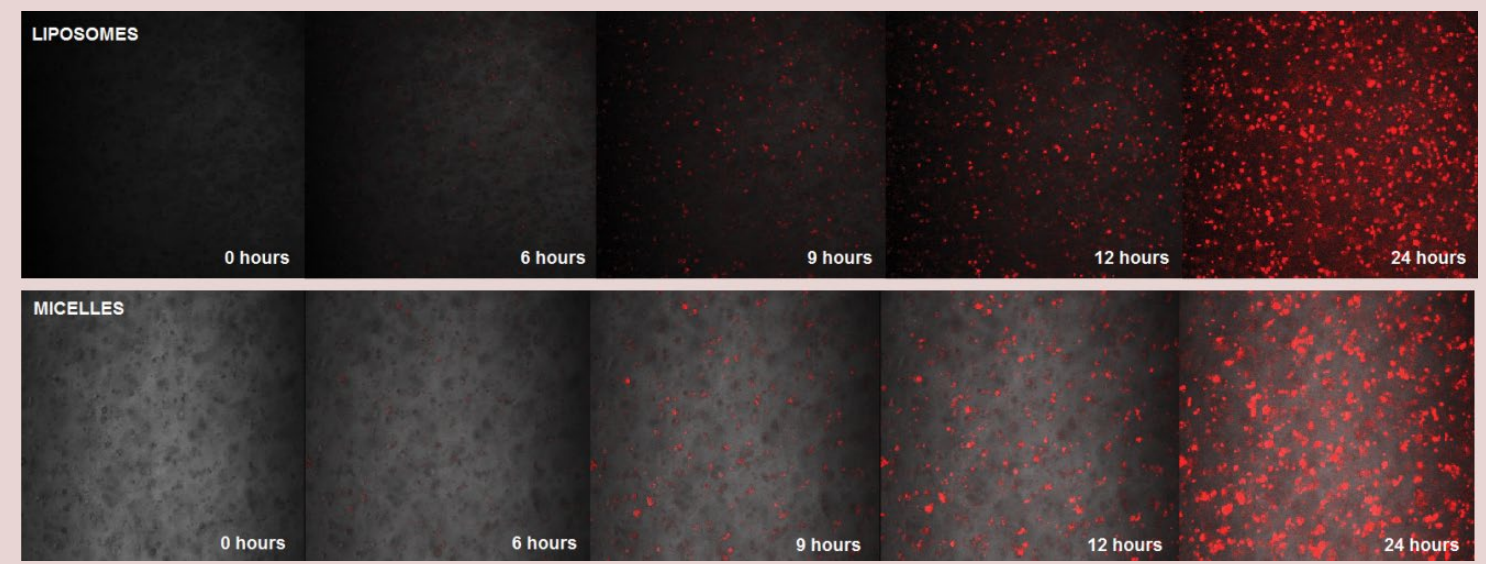

(c)

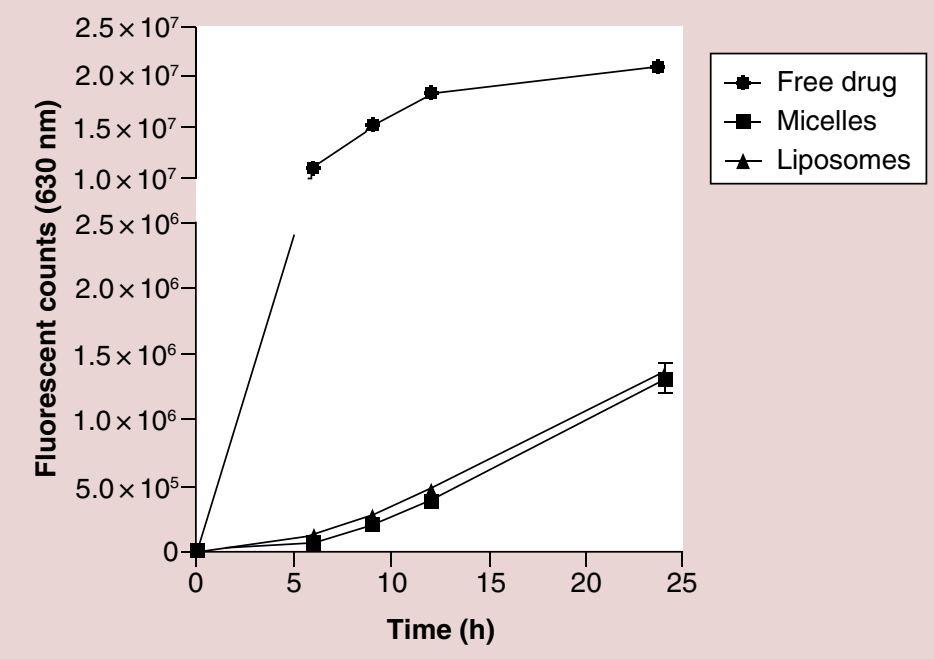

Figure 6. Nanoparticle penetration into the tumoroids. HCT116 cells were seeded in hydrogel-nested artificial cancer masses and left to mature and form aggregates for 7 days. Tumoroids were treated with (A) free Nile red dissolved in DMSO and (B) liposomes and micelles containing Nile red. Images were taken through the center of the artificial cancer mass over a period of $24 \mathrm{~h}$ and (C) fluorescence was quantified using the Image J software.

both models are certainly subject to different conditions. Some examples are changes in gene expression and cell behavior when cells are in contact with each other, drug concentration gradients and differential proliferation patterns in acidic, hypoxic- or nutrientdeprived regions of the constructs (Nyga et al. 2013; Minchinton \& Tannock 2006). 3D cancer models mimic these conditions to make cell behavior more similar to real tumor tissues [10]. However, the differences in drug efficacy between $2 \mathrm{D}$ and $3 \mathrm{D}$ are unpredictable and that highlights the importance of 3D testing during drug development. For example, although Magdeldin et al. reported a two-fold increase of EGFR expression in HT29 cell tumoroids 
compared with monolayers, the anti-EGFR monoclonal antibody cetuximab elicited a weaker inhibitory response in the former [9].

In the present work, the role of the extracellular environment in our tumoroid system was simplified to a compressed collagen matrix. Therefore, any changes in behavior of HCT116 cells are exclusively due to the dense collagen microenvironment of the ACM and the cellular 3D conformation. There is evidence that these parameters are enough to cause radical changes in cell behavior [12].

The greater effect of free AZD6244 in tumoroids compared with monolayers, combined with the superior delivery of the drug observed with the nanoparticles in monolayers, suggest that the reduction in efficacy observed in the nanoparticle treatment of the tumoroids $(60 \%$ for liposomes and $40 \%$ for micelles in monolayers (Figure $3 \mathrm{~B}$ ) vs $26 \%$ inhibition for liposomes and $34 \%$ for micelles in tumoroids [Figure 5C]) was not caused by the drug itself. The most likely scenario to explain the suboptimal efficacy of these liposomes and micelle aggregates in tumoroids is their localization within the ACM. Their diffusion toward the core may be partly limited by their large size, possible interactions with the collagen matrix and the uptake by cancer cell aggregates at the outer boundary of the ACM. The core of the ACM is representative of cancer cells hundreds of micrometers distant from a blood vessel in vivo, to which the drugs have limited access [52]. This hypothesis is supported by our penetration studies (Figure 6). It is worth noting that additional functionalization of the surface with antibodies or PEG, which are traditional surface modifications for in vivo studies, would make these nanoparticles even larger. Furthermore, the presence of serum proteins/glycoproteins would also contribute to nanoparticle agglomeration.

The efficacy of micelles (or micelle aggregates) in tumoroids compared with the liposomes is closer to, and in some cases more effective than, the free drug. While the efficacy of the free drug could be due to a much better penetration, the higher efficacy of micelles could be due to their cationic toxicity (Figure $3 \mathrm{C}$ ), however limited to the periphery. It is also possible that cells in the periphery of the ACM are more metabolically active than those of the core, due to limited diffusion of nutrients across the dense cancer mass. This would favor the toxicity of the nanoparticles that, however remaining in the outer layers of the ACM, have a greater inhibitory effect on metabolic activity.

Molecular weight, solubility, shape and other physicochemical characteristics of a drug influences its diffusion through tissue [52]. It has been suggested for large molecules such as antibodies that size could affect their ability to penetrate 3D models [9,53]. In the case of antibodies, not only their size but their ability to bind to surface receptors could hinder their penetration by sequestration in the outer layers of cells. Similarly, the very uptake of drugs in the periphery of the structure could limit the penetration to deeper layers [52]. Penetration becomes especially important in the case of nanoparticles, which size far exceeds that of drugs, particularly small molecules. Huang et al. demonstrated that different sizes of gold nanoparticles have the ability to penetrate into tumor spheroids in a size-dependent manner. Additionally, the smallest particles $(2-6 \mathrm{~nm})$ were able to reach the nucleus while the largest $(15 \mathrm{~nm})$ were confined to the cytoplasm of cells [13].

\section{Conclusion}

Organic cationic nanoparticles such as GCPQ micelles and DOPE/DC-cholesterol liposomes appear to be excellent carriers for hydrophobic small molecules such as AZD6244. They are successfully internalized by cells grown in monolayers and deliver the drug into the cell more efficiently than the free drug. While AZD6244 as a free drug seems to elicit a higher inhibitory response in tumoroids compared with monolayers, the size and nature of our nanocarriers limit their penetration into the deeper regions of the tumoroid model and therefore hinder their therapeutic capacity. In spite of this, GCPQ micelles showed the highest toxicity in tumoroids, probably due to a higher intrinsic toxicity caused by their size and surface charge. While further work is needed to fully understand their behavior and efficacy in 3D models, these preliminary studies point at GCPQ micelles as the most beneficial formulation for a pharmaceutical scientist. To our knowledge, this is the first time AZD6244 has been tested as a nanoformulation, or indeed any nanocarrier has been tested in a natural scaffold-based 3D cancer model. These results illustrate the efficacy of cationic organic nanocarriers for hydrophobic drug delivery and highlight the importance of accounting for the tumor microenvironment during drug development, particularly in the case of nanoformulations.

\section{Future perspective}

In this work, two of the most promising approaches to the fields of drug development and drug delivery converge: the use of nanocarriers as drug delivery platforms and the use of $3 \mathrm{D}$ in vitro models for drug testing. In the near future, the development of most newly discovered drugs will include testing in 3D models at some point during the preclinical stages and the use of 
animals will be significantly reduced due to this screening process. Similarly, the field of nanomedicine will continue to expand since many initially promising molecules whose progress was interrupted due to adverse biological interactions and physicochemical limitations can be regenerated for new treatment possibilities.

Financial \& competing interests disclosure

V López-Dávila is funded by The Rosetrees Trust (520661) and the Royal Free Charity Trust (519543); I Uchegbu is funded by the EPSRC (EP/L024748/1; EP/G061483/1). The authors have no other relevant affiliations or financial involvement with any organization or entity with a financial interest in or financial conflict with the subject matter or materials discussed in the manuscript apart from those disclosed.
No writing assistance was utilized in the production of this manuscript.

\section{Ethical conduct of research}

The authors state that they have obtained appropriate institutional review board approval or have followed the principles outlined in the Declaration of Helsinki for all human or animal experimental investigations. In addition, for investigations involving human subjects, informed consent has been obtained from the participants involved.

\section{Open access}

This work is licensed under the Creative Commons Attribution 4.0 License. To view a copy of this license, visit http://creativecommons.org/licenses/by/4.0/

Executive summary

DOPE/DC-cholesterol and GCPQ nanoparticles encapsulating AZD6244

- Both nanocarriers were successful in the encapsulation of hydrophobic cargo AZD6244 and show consistent sizes and encapsulation efficiencies.

Nanoparticle toxicity and AZD6244 delivery

- Liposomes and micelles containing AZD6244 deliver the drug more efficiently than a solution of the free drug. This is demonstrated by proliferation assays as well as a reduction in the activity of the target protein.

- Only GCPQ micelles show a moderate toxicity without the cargo, which could be attributed to their strong cationic nature.

Tumoroids as a platform for screening nanoformulations

- The significant differences in treatment efficacy between monolayers and tumoroids suggest that 3D cancer models should be included in the drug development process, particularly in the case of nanoparticles.

Nanoparticle-mediated delivery is hindered by poor penetration into the artificial cancer mass

- Experiments on tumoroids show that the efficacy of large cationic nanoparticles is limited due to a poor diffusion through the cancer mass.

- However, the superior toxicity of GCPQ micelles over the other formulations makes it the most effective on the 3D model.

\section{References}

1 Jemal A, Bray F, Center MM, Ferlay J, Ward E, Forman D. Global cancer statistics. CA Cancer J. Clin. 61(2), 69-90 (2011).

2 Ostman A, Augsten M. Cancer-associated fibroblasts and tumor growth-bystanders turning into key players. Curr. Opin. Genet. Dev. 19(1), 67-73 (2009).

3 Yamada KM, Cukierman E. Modeling tissue morphogenesis and cancer in 3D. Cell 130(4), 601-610 (2007).

4 Bhowmick NA, Neilson EG, Moses HL. Stromal fibroblasts in cancer initiation and progression. Nature 432(7015), 332-337 (2004).

5 Rhee S, Grinnell F. Fibroblast mechanics in 3D collagen matrices. Adv. Drug Deliv. Rev. 59(13), 1299-1305 (2007).

6 Halliwell B. Oxidative stress in cell culture: an underappreciated problem? FEBS Lett. 540(1-3), 3-6 (2003).

7 Voskoglou-Nomikos T, Pater JL, Seymour L. Clinical predictive value of the in vitro cell line, human xenograft, and mouse allograft preclinical cancer models. Clin. Cancer Res. 9(11), 4227-4239 (2003).
8 Sharpless NE, Depinho RA. The mighty mouse: genetically engineered mouse models in cancer drug development. Nat. Rev. Drug Discov. 5(9), 741-754 (2006).

9 Magdeldin T, Lopez-Davila V, Villemant C et al. The efficacy of cetuximab in a tissue-engineered threedimensional in vitro model of colorectal cancer. J. Tissue Eng. 5, 2041731414544183 (2014).

10 Tredan O, Galmarini CM, Patel K, Tannock IF. Drug resistance and the solid tumor microenvironment. J. Natl Cancer Inst. 99(19), 1441-1454 (2007).

11 Mikhail AS, Eetezadi S, Allen C. Multicellular tumor spheroids for evaluation of cytotoxicity and tumor growth inhibitory effects of nanomedicines in vitro: a comparison of docetaxel-loaded block copolymer micelles and Taxotere(R). PLoS ONE 8(4), e62630 (2013).

12 Hickman JA, Graeser R, De Hoogt R et al. Threedimensional models of cancer for pharmacology and cancer cell biology: capturing tumor complexity in vitrolex vivo. Biotechnol. J. 9(9), 1115-1128 (2014).

13 Huang K, Ma H, Liu J et al. Size-dependent localization and penetration of ultrasmall gold nanoparticles in cancer cells, 
multicellular spheroids, and tumors in vivo. ACS Nano 6(5), 4483-4493 (2012).

14 Kunz-Schughart LA, Kreutz M, Knuechel R. Multicellular spheroids: a three-dimensional in vitro culture system to study tumour biology. Int. J. Exp. Pathol. 79(1), 1-23 (1998).

15 Holliday DL, Brouilette KT, Markert A, Gordon LA, Jones JL. Novel multicellular organotypic models of normal and malignant breast: tools for dissecting the role of the microenvironment in breast cancer progression. Breast Cancer Res. 11(1), R3 (2009).

16 Suri S, Schmidt CE. Cell-laden hydrogel constructs of hyaluronic acid, collagen, and laminin for neural tissue engineering. Tissue Eng. A 16(5), 1703-1716 (2010).

17 Gurski LA, Jha AK, Zhang C, Jia X, Farach-Carson MC. Hyaluronic acid-based hydrogels as $3 \mathrm{D}$ matrices for in vitro evaluation of chemotherapeutic drugs using poorly adherent prostate cancer cells. Biomaterials 30(30), 6076-6085 (2009).

18 Christopher S. Szot CFB, Paul Gatenholm, Marissa Nichole Rylander, Joseph W. Freeman. Investigation of cancer cell behavior on nanofibrous scaffolds. Mater. Sci. Eng. C 31(1), 37-42 (2011).

19 Kate PM Ricketts, Umber Cheema, Agata Nyga et al. A 3D In vitro cancer model as a platform for nanoparticle uptake and imaging investigations. Small 10(19), 3954-3961 (2014).

20 Nyga A, Loizidou M, Emberton M, Cheema U. A novel tissue engineered three-dimensional in vitro colorectal cancer model. Acta Biomater. 9(8), 7917-7926 (2013).

21 Lopez-Davila V, Seifalian AM, Loizidou M. Organic nanocarriers for cancer drug delivery. Curr. Opin. Pharmacol. 12(4), 414-419 (2012).

22 Hino M, Ichihara H, Matsumoto Y, Ueoka R. Anti-tumor effects of cationic hybrid liposomes against colon carcinoma along with apoptosis in vitro. Biol. Pharm. Bull. 35(11), 2097-2101 (2012).

23 Erdog A, Putra Limasale YD, Keskin D, Tezcaner A, Banerjee S. In vitro characterization of a liposomal formulation of celecoxib containing 1,2-distearoyl-snglycero-3-phosphocholine, cholesterol, and polyethylene glycol and its functional effects against colorectal cancer cell lines. J. Pharm. Sci. 102(10), 3666-3677 (2013).

24 Casado A, Sagrista ML, Mora M. Formulation and in vitro characterization of thermosensitive liposomes for the delivery of irinotecan. J. Pharm. Sci. 103(10), 3127-3138 (2014).

25 Guo S, Huang L. Nanoparticles containing insoluble drug for cancer therapy. Biotechnol. Adv. 32(4), 778-788 (2014).

26 Zhang Y, Li H, Sun J et al. DC-Chol/DOPE cationic liposomes: a comparative study of the influence factors on plasmid pDNA and siRNA gene delivery. Int. J. Pharm. 390(2), 198-207 (2010).

27 Yang S, Chen J, Zhao D, Han D, Chen X. Comparative study on preparative methods of DC-Chol/DOPE liposomes and formulation optimization by determining encapsulation efficiency. Int. J. Pharm. 434(1-2), 155-160 (2012).

28 Ciani L, Ristori S, Salvati A, Calamai L, Martini G. DOTAP/DOPE and DC-Chol/DOPE lipoplexes for gene delivery: zeta potential measurements and electron spin resonance spectra. Biochim. Biophys. Acta 1664(1), 70-79 (2004).

29 Qu X, Khutoryanskiy VV, Stewart A et al. Carbohydratebased micelle clusters which enhance hydrophobic drug bioavailability by up to 1 order of magnitude. Biomacromolecules 7(12), 3452-3459 (2006).

30 Siew A, Le H, Thiovolet M, Gellert P, Schatzlein A, Uchegbu I. Enhanced oral absorption of hydrophobic and hydrophilic drugs using quaternary ammonium palmitoyl glycol chitosan nanoparticles. Mol. Pharm. 9(1), 14-28 (2012).

31 Lalatsa A, Garrett NL, Ferrarelli T, Moger J, Schatzlein AG, Uchegbu IF. Delivery of peptides to the blood and brain after oral uptake of quaternary ammonium palmitoyl glycol chitosan nanoparticles. Mol. Pharm. 9(6), 1764-1774 (2012).

32 Pelaz B, Charron G, Pfeiffer C et al. Interfacing engineered nanoparticles with biological systems: anticipating adverse nano-bio interactions. Small 9(9-10), 1573-1584 (2013).

33 Dokka S, Toledo D, Shi X, Castranova V, Rojanasakul Y. Oxygen radical-mediated pulmonary toxicity induced by some cationic liposomes. Pharm. Res. 17(5), 521-525 (2000).

34 Lee WL, Guo WM, Ho VH et al. Inhibition of 3-D tumor spheroids by timed-released hydrophilic and hydrophobic drugs from multilayered polymeric microparticles. Small 10(19), 3986-3996 (2014).

35 Oishi M, Nagasaki Y, Nishiyama $\mathrm{N}$ et al. Enhanced growth inhibition of hepatic multicellular tumor spheroids by lactosylated poly(ethylene glycol)-siRNA conjugate formulated in PEGylated polyplexes. ChemMedChem 2(9), 1290-1297 (2007).

36 Perche F, Patel NR, Torchilin VP. Accumulation and toxicity of antibody-targeted doxorubicin-loaded PEG-PE micelles in ovarian cancer cell spheroid model. J. Control. Release 164(1), 95-102 (2012).

37 Mozafari MR. Nanoliposomes: preparation and analysis. Methods Mol. Biol. 605, 29-50 (2010).

38 RA Brown MW, Chuo CB, Cheema U, Nazhat SN. Ultrarapid engineering of biomimetic materials and tissues: fneabrication of nano- and microstructures by plastic compression. Adv. Funct. Mater. 15(11), 1762-1770 (2005).

39 Ciombor KK, Bekaii-Saab T. Selumetinib for the treatment of cancer. Expert Opin. Invest. Drugs 24(1), 111-123 (2015).

40 Basu S, Harfouche R, Soni S, Chimote G, Mashelkar RA, Sengupta S. Nanoparticle-mediated targeting of MAPK signaling predisposes tumor to chemotherapy. Proc. Natl Acad. Sci. USA 106(19), 7957-7961 (2009).

41 Kang SH, Cho HJ, Shim G et al. Cationic liposomal codelivery of small interfering RNA and a MEK inhibitor for enhanced anticancer efficacy. Pharm. Res. 28(12), 3069-3078 (2011).

42 Lin J, Zhang H, Chen Z, Zheng Y. Penetration of lipid membranes by gold nanoparticles: insights into cellular uptake, cytotoxicity, and their relationship. ACS Nano 4(9), 5421-5429 (2010).

43 Pisani M, Mobbili G,, Paolo Bruni. Neutral Liposomes and DNA transfection. In: Non-Viral Gene Therapy. Yuan X-B (Ed.). Intech, Rijeka, Croatia (2011). 
44 Ichihara H, Hino M, Umebayashi M, Matsumoto Y, Ueoka R. Intravenous injection of hybrid liposomes suppresses the liver metastases in xenograft mouse models of colorectal cancer in vivo. Eur. J. Med. Chem. 57, 143-148 (2012).

45 Murrell MP, Voituriez R, Joanny JF, Nassoy P, Sykes C, Gardel ML. Liposome adhesion generates traction stress. Nat. Phys. 10 (2), 163-169 (2014).

46 Shah V, Taratula O, Garbuzenko OB et al. Genotoxicity of different nanocarriers: possible modifications for the delivery of nucleic acids. Curr. Drug Discov. Technol. 10(1), 8-15 (2013).

47 Andar AU, Hood RR, Vreeland WN, Devoe DL, Swaan PW. Microfluidic preparation of liposomes to determine particle size influence on cellular uptake mechanisms. Pharm. Res. 31(2), 401-413 (2014).

48 Polo ML, Arnoni MV, Riggio M, Wargon V, Lanari C, Novaro V. Responsiveness to PI3K and MEK inhibitors in breast cancer. Use of a 3D culture system to study pathways related to hormone independence in mice. PLoS ONE 5(5), e10786 (2010).
49 Caputo E, Miceli R, Motti ML et al. AurkA inhibitors enhance the effects of B-RAF and MEK inhibitors in melanoma treatment. J. Transl. Med. 12, 216 (2014).

50 Ferguson J, Arozarena I, Ehrhardt M, Wellbrock C. Combination of MEK and SRC inhibition suppresses melanoma cell growth and invasion. Oncogene 32(1), 86-96 (2013).

51 Chambers KF, Mosaad EM, Russell PJ, Clements JA, Doran MR. 3D Cultures of prostate cancer cells cultured in a novel high-throughput culture platform are more resistant to chemotherapeutics compared with cells cultured in monolayer. PLoS ONE 9(11), e111029 (2014).

52 Minchinton AI, Tannock IF. Drug penetration in solid tumours. Nat. Rev. Cancer 6(8), 583-592 (2006).

53 Xiang X, Phung Y, Feng M et al. The development and characterization of a human mesothelioma in vitro $3 \mathrm{D}$ model to investigate immunotoxin therapy. PLoS ONE 6(1), e14640 (2011). 